Pacific Journal of Mathematics

ENVELOPES OF HOLOMORPHY OF HARTOGS AND 


\section{ENVELOPES OF HOLOMORPHY OF HARTOGS AND CIRCULAR DOMAINS}

\section{Enrico Casadio Tarabusi and Stefano Trapani}

Conditions are given for the envelope of holomorphy of a Hartogs or circular domain in $C^{n}$ to be univalent, together with its explicit construction. The noneliminability of the assumptions is shown by counterexamples.

0. Introduction. In this paper we consider the classical problem of the univalence and description of the envelope of holomorphy $E(\Omega)$ of a domain $\Omega$ in $\mathbf{C}^{n}$ (see [Cm] for a survey): unless otherwise stated, $n$ will be assumed to be $\geq 2$. We take into consideration the classes of Hartogs and of circular domains.

The Hartogs case has been extensively studied in the past (see, e.g., [V]). We deepen its investigation proving some stronger results, particularly for domains having "connected vertical sections" (see $\S 2$ for definitions), which include the ones previously considered. In Theorem 2.4, for instance, we give necessary and sufficient conditions for the univalence of $E(\Omega)$, along with its description whenever such conditions hold.

Using the achievements of the Hartogs case, we are able to obtain similar ones especially for circular domains having "connected linear sections" with complex lines through the origin. We do this by observing that Hartogs domains disjoint from their hyperplane of symmetry and circular domains which do not intersect a hyperplane through the origin correspond to one another through a biholomorphism $h$ of $\mathbf{C}^{n-1} \times \mathbf{C}^{*}$ onto itself; and inferring the general case from it.

With suitable examples we show that the hypothesis of connected (vertical or linear) sections cannot be dropped from any of our main statements.

Finally we give an alternative interpretation of the results of the circular case in terms of fiber bundles over the projective space $\mathbf{P}^{n-1}(\mathbf{C})$.

The authors wish to thank the referee for his helpful suggestions.

1. Preliminary definitions and results. The envelope of holomorphy of a domain $\Omega$ in $\mathbf{C}^{n}$ (with $n \geq 1$ ) will be denoted by $E(\Omega)=$ $(E(\Omega), \eta, \lambda)$, where $\lambda: \Omega \rightarrow E(\Omega), \eta: E(\Omega) \rightarrow \mathbf{C}^{n}$ are the canonical 
maps. We say that $E(\Omega)$ is schlicht if $\eta$ is one-to-one; in this case $E(\Omega)$ will be identified with $\eta(E(\Omega))$.

We will say that a pair $(D ; \Omega)$ of domains in $\mathbf{C}^{n}$ (with $n \geq 1$ ) has the Runge property if $D$ contains $\Omega$ and if every holomorphic function on $\Omega$ is approximable in the compact-open topology by the restrictions to $\Omega$ of holomorphic functions on $D$ : if both $D, \Omega$ are domains of holomorphy this is the usual definition of Runge pair.

LEMMA 1.1. If the pair $(D ; \Omega)$ has the Runge property and $E(D)$ is schlicht, then $E(\Omega)$ is schlicht and $(E(D) ; E(\Omega))$ is a Runge pair.

Proof. Obviously $(E(D) ; \Omega)$ has the Runge property. Let $\left(K_{\nu}\right)_{\nu \in \mathbf{N}}$ be a normal exhaustion (see [GF, pp. 46-47] for terminology) of $\Omega$ by compact sets, and let $\Omega^{\prime}$ be the connected component containing $\Omega$ of the union of the interiors of

$$
\widehat{K}_{\nu}^{E(D)}=\left\{\mathbf{x} \in E(D):|f(\mathbf{x})| \leq \max _{K_{\nu}}|f| \text { for every } f \in \mathscr{O}(E(D))\right\} .
$$

So $\Omega^{\prime}$ is a domain of holomorphy, it being a component of the union of an increasing sequence of open sets of holomorphy in $\mathbf{C}^{n}$. If the sequence $\left(f_{k}\right)_{k \in \mathbf{N}} \subset \mathscr{O}(E(D))$ converges on $\Omega$ to a given $f \in \mathscr{O}(\Omega)$, then it is a Cauchy sequence in every $K_{\nu}$, so in every $\widehat{K}_{\nu}^{E(D)}$. It therefore converges on $\Omega^{\prime}$ to an $f^{\prime} \in \mathscr{O}\left(\Omega^{\prime}\right)$ which coincides with $f$ on $\Omega$.

LEMMA 1.2. Let $D$ be a domain of holomorphy in $\mathrm{C}^{n}, \Omega$ be $a$ subdomain of $D, f$ be a holomorphic function on $D$ not vanishing identically, $S=\{f=0\} \subset D$. Then $E(\Omega \backslash S)$ is schlicht if and only if $E(\Omega)$ is; in this case $E(\Omega \backslash S)=E(\Omega) \backslash S$.

Proof. The "if" part and the final equality of the statement easily follow from [GrR, Satz 7 p. 165].

Assume that $E(\Omega \backslash S)$ is schlicht. We first prove that the interior $\Omega^{\prime}$ of $E(\Omega \backslash S) \cup S$ is a domain of holomorphy. Let $\left(E\left(\Omega^{\prime}\right), \eta, \lambda\right)$ be the envelope of holomorphy of $\Omega^{\prime}$, and let $\widetilde{S}=\{\tilde{f}=0\} \subset E\left(\Omega^{\prime}\right)$, where $\tilde{f}$ is the holomorphic extension to $E\left(\Omega^{\prime}\right)$ of $\left.f\right|_{\Omega^{\prime}}$. Thus, since $f$ is defined on all of $\eta\left(E\left(\Omega^{\prime}\right)\right)$, we have $\tilde{f}=f \circ \eta$, so $\tilde{S}=\eta^{-1}(S) ?$ Again by [GrR, Satz 7 p. 165] we gather that $\left(E\left(\Omega^{\prime}\right) \backslash \widetilde{S}, \eta, \lambda\right)$ is the envelope of holomorphy of $\Omega^{\prime} \backslash S=E(\Omega \backslash S)$, so $\eta\left(E\left(\Omega^{\prime}\right) \backslash \widetilde{S}\right)=$ $\Omega^{\prime} \backslash S$, because $\Omega^{\prime} \backslash S$ is already a domain of holomorphy. Hence $\eta\left(E\left(\Omega^{\prime}\right)\right) \backslash S=\Omega^{\prime} \backslash S$ (recall that $\widetilde{S}=\eta^{-1}(S)$ ), and, by definition of 
$\Omega^{\prime}, \eta\left(E\left(\Omega^{\prime}\right)\right)=\Omega^{\prime}$. If $\Delta$ is a subdomain of $D$ for which there exists a connected component $\Delta^{\prime}$ of $\Delta \cap \Omega^{\prime}$ so that the restriction to $\Delta^{\prime}$ of every holomorphic function on $\Omega^{\prime}$ extends holomorphically to $\Delta$, then $\Delta$ is contained in $\eta\left(E\left(\Omega^{\prime}\right)\right)$, and so in $\Omega^{\prime}$, therefore $\Omega^{\prime}$ is a domain of holomorphy.

Thus we can assume $D=\Omega^{\prime}$ without loss of generality. Let $\left(S_{j}\right)_{j \in J}$ be the family of irreducible components of $S$, let $J_{0}=\left\{j \in J: S_{j}\right.$ intersects $\Omega\}$, and for $j \in J_{0}$ let $D_{j}=D \backslash\left(\bigcup_{k \in J ; k \neq j} S_{k}\right)$. Since, for $j \in J_{0}, S_{j} \cap \Omega$ has codimension 1 in $D$, whereas $\operatorname{sing}(S)$, the singular locus of $S$, has codimension at least 2 in $D$, then $S_{j} \cap \Omega$ contains smooth points of $S$, so $S_{j} \cap D_{j} \cap \Omega$ is nonempty for every $j \in J_{0}$.

The restriction to $\Omega \backslash S$ of $g \in \mathscr{O}(\Omega)$ extends holomorphically to $E(\Omega \backslash S)=D \backslash S=D_{j} \backslash S_{j}$ for each $j \in J_{0}$. Moreover, since $S_{j} \cap D_{j} \cap \Omega \neq \varnothing$, then $\left.g\right|_{\Omega \backslash S}$ also extends holomorphically to a small neighborhood of some point of the irreducible hypersurface $D_{j} \cap S_{j}$, therefore it extends to a holomorphic function $g_{j}$ on $D_{j}$ for each $j \in J_{0}$. Now, given distinct $j, k$ in $J_{0}$, we have $D_{j} \cap D_{k}=$ $D \backslash S=E(\Omega \backslash S)$, therefore $g_{j} \equiv g_{k}$ on $D_{j} \cap D_{k}$. So $\left.g\right|_{\Omega \backslash S}$ extends holomorphically to $\bigcup_{j \in J_{0}} D_{j}$, which contains $D \backslash(T \cup \operatorname{sing}(S))$, where $T=\bigcup_{j \in J \backslash J_{0}} S_{j}$. Since $\operatorname{sing}(S)$ has codimension at least 2 in $D$, then $\left.g\right|_{\Omega \backslash S}$ extends holomorphically to $D \backslash T$. But $\Omega$ is contained in the domain of holomorphy $D \backslash T$; hence $D \backslash T=E(\Omega)$, which is schlicht.

For brevity of exposition, the locutions "plurisubharmonic" and "plurisuperharmonic" will henceforth stand for "plurisubharmonic or $\equiv-\infty$ " and "plurisuperharmonic or $\equiv+\infty$ ", respectively.

LEMMA 1.3. Assume $\boldsymbol{\Omega}$ is a domain in $\mathbf{C}^{n}$ with schlicht envelope of holomorphy $E(\Omega)$. Let $u: \Omega \rightarrow[-\infty,+\infty[$ be an upper semicontinuous function, and

$$
\begin{array}{r}
F_{u}=\{v: E(\Omega) \rightarrow[-\infty,+\infty[: v \text { is plurisubharmonic on } E(\Omega) \\
\text { and } v \leq u \text { on } \Omega\} .
\end{array}
$$

Then $\mathscr{F}_{u}$ has a maximum element $u_{*}$.

Proof. By $[\mathrm{V}, \S \S 10.3-4$ p. 74$]$ we only need to show that $\mathscr{F}_{u}$ is locally uniformly bounded from above. For $k \in \mathbf{N}$, set $\Omega_{k}=\{u<k\}$ : thus $\Omega=\bigcup_{k \in N} \Omega_{k}$. Let $\Omega_{k}^{\prime}$ be the interior of the intersection of all the open sets of holomorphy containing $\Omega_{k}$ : by [H, Corollary 2.5 .7 p. 40] (which is stated for domains, but whose proof works for open sets as 
well) $\Omega_{k}^{\prime}$ is itself an open set of holomorphy in $\mathbf{C}^{n}$. Then $U_{k \in \mathbf{N}} \Omega_{k}^{\prime}$ is an open set of holomorphy, so it coincides with $E(\Omega)$. Given $v \in \mathscr{F}_{u}$, for each $k \in \mathbf{N},\{v<k\}$ is an open set of holomorphy containing $\Omega_{k}$, therefore it contains $\Omega_{k}^{\prime}$; that is, $\mathscr{F}_{u}$ is uniformly bounded from above by $k$ on the open set $\Omega_{k}^{\prime}$.

Let $\Omega$ be as above. If $u: \Omega \rightarrow[0,+\infty[$ is upper semicontinuous, let $u_{(*)}=e^{(\log u)_{*}}$. Dually, if $\left.\left.u: \Omega \rightarrow\right]-\infty,+\infty\right]$ is lower semicontinuous, set $u^{*}=-(-u)_{*}$, and if $\left.\left.u: \Omega \rightarrow\right] 0,+\infty\right]$ is lower semicontinuous set $u^{(*)}=e^{(\log u)^{*}}$.

Two more conventions: (1) the norm $\|\cdot\|$ in $\mathbf{C}^{n}$ is assumed to be the euclidean one; and (2) when the argument of a complex number appears in an expression, it will always mean one of its choices.

2. Hartogs domains. A domain $\Omega$ in $\mathbf{C}^{n}$ is a Hartogs domain in $w$ if $(\mathbf{z}, w)=\left(z_{1}, \ldots, z_{n-1}, w\right) \in \Omega$ implies $\left(\mathbf{z}, e^{i \theta} w\right) \in \Omega$ for any $\theta \in \mathbf{R}$. Let $\pi: \mathbf{C}^{n} \rightarrow \mathbf{C}^{n-1}$ be the projection $\pi(\mathbf{z}, w)=\mathbf{z}$. If $\Omega$ is a Hartogs domain (in $w$, unless otherwise stated), define $a, b: \pi(\Omega) \rightarrow[0,+\infty]$ by

$$
a(\mathbf{z})=\inf _{(\mathbf{z}, w) \in \Omega}|w|, \quad b(\mathbf{z})=\sup _{(\mathbf{z}, w) \in \Omega}|w| ;
$$

thus $a$ is upper semicontinuous and $b$ is lower semicontinuous. A Hartogs domain $\Omega$ will be said to have connected vertical sections if for each $\mathbf{z} \in \pi(\Omega)$ the set

$$
A_{\mathbf{z}}=\{w \in \mathbf{C}:(\mathbf{z}, w) \in \Omega\}
$$

is connected; if $A_{\mathbf{z}}$ is a disk for all $\mathbf{z} \in \pi(\Omega)$ then $\Omega$ is called complete.

Note that if $\Omega$ is a Hartogs domain having schlicht envelope of holomorphy then $E(\Omega)$ is itself Hartogs (cf. [V, $\S 20.5$ p. 180]).

Proposition 2.1. Let $\Omega$ be a Hartogs domain.

(1) If $\Omega$ intersects $\{w=0\}$, then $\Omega$ is a domain of holomorphy if and only if $\Omega$ is complete, $\pi(\Omega)$ is a domain of holomorphy, and $\log b$ is plurisuperharmonic.

(2) If $\Omega$ does not intersect $\{w=0\}$ and has connected vertical sections, then $\Omega$ is a domain of holomorphy if and only if $\pi(\Omega)$ is a domain of holomorphy and $\log a,-\log b$ are plurisubharmonic.

Proof. (1) If $\Omega$ is a domain of holomorphy, its completeness immediately follows from the fact that each $(\mathbf{z}, w) \in \pi(\Omega) \times \mathbf{C}$ such 
that $|w|<b(z)$ is in $\eta(E(\Omega))$ by $[\mathbf{V}, \S 15.2$ p. 125]. The remainder of case (1) now follows from [V, $\S 19.4$ p. 174].

(2) Let $(P, H)$ be a general Hartogs figure in $\mathbf{C}^{n-1}$ (see [GF, Definition II.1.1 p. 29]; $H$ is not necessarily a Hartogs domain) such that $H \subset \pi(\Omega)$. Set

$$
\begin{aligned}
& \boldsymbol{\Omega}_{1}=\{(\mathbf{z}, w) \in H \times \mathbf{C}:|w|<b(\mathbf{z})\}, \\
& \boldsymbol{\Omega}_{2}=\{(\mathbf{z}, w) \in H \times \mathbf{C}:|w|<1 / a(\mathbf{z})\},
\end{aligned}
$$

and, if $\phi: \mathbf{C}^{n} \backslash\{w=0\} \rightarrow \mathbf{C}^{n}$ is given by $\phi(\mathbf{z}, w)=(\mathbf{z}, 1 / w)$,

$$
\Omega_{3}=\Omega_{1} \cap \phi\left(\Omega_{2} \backslash\{w=0\}\right)=\Omega \cap(H \times C) .
$$

From the Laurent series expansion of $f \in \mathscr{O}\left(\Omega_{3}\right)$ in the $w$ variable, centered at $w=0$, we derive that $f=f_{1}+f_{2} \circ \phi$ with $f_{j} \in \mathscr{O}\left(\Omega_{j}\right)$, $j=1$, 2. By [VS, Teorema p. 191] $E\left(\Omega_{1}\right), E\left(\Omega_{2}\right)$ are schlicht and respectively equal to

$$
\begin{aligned}
& \left\{(\mathbf{z}, w) \in P \times \mathbf{C}:|w|<\left(\left.b\right|_{H}\right)^{(*)}(\mathbf{z})\right\} \\
& \left\{(\mathbf{z}, w) \in P \times \mathbf{C}:|w|<\left(1 /\left.a\right|_{H}\right)^{(*)}(\mathbf{z})=1 /\left(\left.a\right|_{H}\right)_{(*)}(\mathbf{z})\right\} ;
\end{aligned}
$$

moreover the inequality $\left(\left.a\right|_{H}\right)_{(*)}<\left(\left.b\right|_{H}\right)^{(*)}$ holds on $H$ : since $\log \left(\left.a\right|_{H}\right)_{(*)},-\log \left(\left.b\right|_{H}\right)^{(*)}$ are plurisubharmonic, it also holds on $P$. If $\Omega$ is a domain of holomorphy, then

$$
\begin{aligned}
E\left(\Omega_{3}\right) & =E\left(\Omega_{1}\right) \cap \phi\left(E\left(\Omega_{2}\right) \backslash\{w=0\}\right) \\
& =\left\{(\mathbf{z}, w) \in P \times \mathbf{C}:\left(\left.a\right|_{H}\right)_{(*)}(\mathbf{z})<|w|<\left(\left.b\right|_{H}\right)^{(*)}(\mathbf{z})\right\}
\end{aligned}
$$

is still contained in $\Omega$, therefore $P \subset \pi(\Omega)$; thus $\pi(\Omega)$ is a domain of holomorphy by [GF, Definition II.2.1 p. 35 and Theorem II.6.2 p. 51].

The conclusion is similar to that in case (1).

EXAMPLE 2.2. A Hartogs domain of holomorphy $\Omega$ in $\mathbf{C}^{3}$ such that $\pi(\Omega)$ is not a domain of holomorphy.

Let

$$
\begin{aligned}
\mathbf{\Omega}=\left\{(\mathbf{z}, w) \in \mathbf{C}^{3}: 0<\log \left|z_{1}\right|<1,\right. \\
\left.\quad \log |w|<\arg z_{1}<\log |w|+1,\left|z_{2}\right|<\arg z_{1}<4 \pi\right\} .
\end{aligned}
$$

For each $(\mathbf{z}, w) \in \Omega$, such choice of $\arg z_{1}$ is obviously unique, and depends continuously on $(\mathbf{z}, w) \in \Omega$. It is trivial to see that $\Omega$ is nonempty and connected; since each inequality defining $\Omega$ can be written as $\psi<0$, where $\psi$ is a plurisubharmonic function on a 
neighborhood of the closure of $\Omega$, we gather that $\Omega$ is a domain of holomorphy. Now

$$
\pi(\Omega)=\left\{\mathbf{z} \in \mathbf{C}^{2}: 1<\left|z_{1}\right|<e,\left|z_{2}\right|<\arg z_{1}<4 \pi\right\}
$$

is a complete Hartogs domain in $z_{2}$, but the corresponding $\log b$ is not superharmonic: in fact $b\left(z_{1}\right)$ equals the choice of $\arg z_{1}$ in $[2 \pi, 4 \pi[$, thus it takes a minimum value $2 \pi$ without being constant. By Proposition 2.1, $\pi(\Omega)$ is not a domain of holomorphy.

REMARK 2.3. Projecting the above defined $\Omega$ onto the $\left(z_{1}, w\right)$ plane we obtain a Hartogs domain of holomorphy

$$
\begin{aligned}
& \left\{\left(z_{1}, w\right) \in \mathbf{C}^{2}: 0<\log \left|z_{1}\right|<1, \quad \log |w|<\arg z_{1}<\log |w|+1,\right. \\
& \left.0<\arg z_{1}<4 \pi\right\}
\end{aligned}
$$

which is not with connected vertical sections. As done in Example 2.2, one easily checks that neither of the corresponding $\log a,-\log b$ is subharmonic.

We are now ready to state the main result concerning Hartogs domains.

THEOREM 2.4. Let $\Omega$ be a Hartogs domain with connected vertical sections. Two possibilities occur:

(1) $\Omega$ intersects $\{w=0\}$ : then $E(\Omega)$ is schlicht if and only if $E(\pi(\Omega)$ ) is schlicht; in this case $E(\pi(\Omega))=\pi(E(\Omega))$, the pair $(E(\pi(\Omega)) \times \mathbf{C} ; E(\Omega))$ is Runge and

$$
E(\Omega)=\left\{(\mathbf{z}, w) \in E(\pi(\Omega)) \times \mathbf{C}:|w|<b^{(*)}(\mathbf{z})\right\} ;
$$

(2) $\Omega$ does not intersect $\{w=0\}$ : then $E(\Omega)$ is schlicht and has connected vertical sections if and only if $E(\pi(\Omega))$ is schlicht; in this case $E(\pi(\Omega))=\pi(E(\Omega))$, the pair $\left(E(\pi(\Omega)) \times \mathbf{C}^{*} ; E(\Omega)\right)$ is Runge and

$$
E(\Omega)=\left\{(\mathbf{z}, w) \in E(\pi(\Omega)) \times \mathbf{C}^{*}: a_{(*)}(\mathbf{z})<|w|<b^{(*)}(\mathbf{z})\right\} .
$$

Proof. Assume $E(\Omega)$ is schlicht and has connected vertical sections (in case (1), the former assumption implies the latter by Proposition 2.1). For $f \in \mathscr{O}(\pi(\Omega))$, the analytic continuation of $f \circ \pi$ to $E(\Omega)$ does not depend on $w$, so it naturally gives $\tilde{f} \in \mathscr{O}(\pi(E(\Omega)))$ that extends $f$. But $\pi(E(\Omega))$ is a domain of holomorphy by Proposition 2.1 , so $\pi(E(\Omega))=E(\pi(\Omega))$.

Suppose $E(\pi(\Omega))$ is schlicht. The coefficients of the Laurent expansion of $f \in \mathscr{O}(\Omega)$ in $w$ extend to $E(\pi(\Omega))$. In case (2) the pair 
$\left(E(\pi(\Omega)) \times \mathrm{C}^{*} ; \Omega\right)$ has the Runge property. While in case (1) the coefficients of negative degree vanish identically on $\pi(\Omega \cap\{w=0\})$, so on $E(\pi(\Omega))$; therefore the Laurent series is in fact a Taylor series in $w$, and $(E(\pi(\Omega)) \times \mathbf{C} ; \Omega)$ has the Runge property. By Lemma 1.1, $E(\Omega)$ is schlicht in both cases, and the pair $(E(\pi(\Omega)) \times \mathbf{C} ; E(\Omega))$ in case (1), or $\left(E(\pi(\Omega)) \times \mathbf{C}^{*} ; E(\Omega)\right)$ in case (2), is Runge. If $\mathbf{z} \in \pi(E(\Omega))$, each holomorphic function on $\widetilde{A_{\mathbf{z}}}=\{w \in \mathbf{C}:(\mathbf{z}, w) \in E(\Omega)\}$ extends to a holomorphic function on the domain of holomorphy $E(\Omega)$ by Cartan's theorem B (see, e.g., [GuR, Theorem VIII.18.(2) p. 245]). Hence, in case $(2)$, the pair $\left(\mathbf{C}^{*} ; \widetilde{A_{\mathbf{z}}}\right)$ is Runge because $\left(E(\pi(\Omega)) \times \mathbf{C}^{*} ; E(\Omega)\right)$ is: so $\widetilde{A}_{\mathbf{z}}$ must be connected.

By Proposition $2.1, \widetilde{\Omega}=\left\{(\mathbf{z}, w) \in E(\pi(\Omega)) \times \mathbf{C}:|w|<b^{(*)}(\mathbf{z})\right\}$ is a domain of holomorphy containing $\Omega$, so $\widetilde{\Omega}$ contains $E(\Omega)=$ $\{(\mathbf{z}, w) \in E(\pi(\Omega)) \times \mathbf{C}:|w|<\tilde{b}(z)\}$, where $\tilde{b} \geq b$ on $\pi(\Omega)$ and $\log \tilde{b}$ is plurisuperharmonic on $E(\pi(\Omega))$. Therefore $\tilde{b} \geq b^{(*)}$, whence $\widetilde{\Omega} \subset E(\Omega)$, and (1) is completely proved. The rest of $(2)$ is taken care of similarly.

Since every domain in $\mathbf{C}$ is a domain of holomorphy we deduce

COROLLARY 2.5. If the Hartogs domain $\Omega \subset \mathrm{C}^{2}$ has connected vertical sections, then $E(\Omega)$ is schlicht and has connected vertical sections, and $\pi(E(\Omega))=\pi(\Omega)$.

REMARK 2.6. If $\Omega$ is the same as in Example 2.2, then the Hartogs domain

$$
D=\left\{(\mathbf{z}, w) \in \Omega: \arg z_{1}-2<\left|z_{2}\right|\right\}
$$

has connected vertical sections, but its schlicht envelope of holomorphy does not. In fact $E(D)=\Omega$ : each $f \in \mathscr{O}(D)$ extends to an $\tilde{f} \in \mathscr{O}(\Omega)$ through the formula:

$$
\begin{aligned}
& \tilde{f}(\mathbf{z}, w)=\frac{1}{2 \pi i} \int_{|\zeta|=\arg z_{1}-1} \frac{f\left(z_{1}, \zeta, w\right)}{\zeta-z_{2}} d \zeta \\
& \text { for }(\mathbf{z}, w) \in \Omega \text { with }\left|z_{2}\right|<\arg z_{1}-1
\end{aligned}
$$

(the choice of $\arg z_{1}$ being the same as in Example 2.2).

So by Theorem 2.4 the envelope of holomorphy of the domain

$$
\begin{aligned}
\pi(D)=\left\{\mathbf{z} \in \mathbf{C}^{2}:\right. & 1<\left|z_{1}\right|<e, \\
& \left.\left|z_{2}\right|<\arg z_{1}<\left|z_{2}\right|+2, \arg z_{1}<4 \pi\right\}
\end{aligned}
$$


is not schlicht: notice that $\pi(D)$, which essentially coincides with $\mathrm{P}$. Thullen's classical example [T, $\S 4$ p. 76], is itself a Hartogs domain in $z_{2}$ intersecting $\left\{z_{2}=0\right\}$. By Lemma 1.2,E( $\left.\pi(D) \backslash\left\{z_{2}=0\right\}\right)$ is not schlicht either.

In the following proposition we make no assumption of connected vertical sections.

Proposition 2.7. If $\Omega$ is a Hartogs domain such that $(\mathbf{z}, w) \in \Omega$ implies $(\mathbf{z}, 0) \in \Omega$, then the same conclusions hold as in (1) of Theorem 2.4.

Proof. Let $\delta: \Omega \rightarrow] 0,+\infty]$ be given by

$$
\delta(\mathbf{z}, w)=\sup \{\varepsilon>0:(\mathbf{z}, \alpha w) \in \Omega
$$

for each $\alpha \in \mathbf{C}$ such that $1 \leq|\alpha|<1+\varepsilon\}$.

Since $\Omega$ is open, the function $\delta$ is lower semicontinuous, so, for $\varepsilon>0$, the set $\Omega_{\varepsilon}=\{(\mathbf{z}, w) \in \Omega: \delta(\mathbf{z}, w)>\varepsilon\}$ is itself open. Fixed $f \in \mathscr{O}(\Omega)$, it is immediate to see that $f_{\varepsilon} \in \mathscr{O}\left(\Omega_{\varepsilon}\right)$ given by

$$
f_{\varepsilon}(\mathbf{z}, w)=\frac{1}{2 \pi i} \int_{|t|=1+\varepsilon} \frac{f(\mathbf{z}, t w)}{t-1} d t \quad \text { for every }(\mathbf{z}, w) \in \Omega_{\varepsilon}
$$

can be expanded into a series, convergent in the compact-open topology, as

$$
f_{\varepsilon}(\mathbf{z}, w)=\sum_{k=0}^{\infty} \frac{1}{2 \pi i} \int_{|t|=1+\varepsilon} \frac{f(\mathbf{z}, t w)}{t^{k+1}} d t \quad \text { for every }(\mathbf{z}, w) \in \Omega_{\varepsilon} .
$$

On the other hand

$$
f(\mathbf{z}, w)=\sum_{k=0}^{\infty} a_{k}(z) w^{k} \quad \text { for }(\mathbf{z}, w) \in D,
$$

where $a_{k} \in \mathscr{O}(\pi(\Omega))$ and

$$
D=\{(\mathbf{z}, w) \in \Omega:(\mathbf{z}, \alpha w) \in \Omega \text { for each } \alpha \in \mathbf{C} \text { such that }|\alpha| \leq 1\} ;
$$

in fact, in our hypotheses, $\pi(D)=\pi(\Omega)$. It is straightforward to check that

$$
\frac{1}{2 \pi i} \int_{|t|=1+\varepsilon} \frac{f(\mathbf{z}, t w)}{t^{k+1}} d t=a_{k}(z) w^{k}
$$

for every $(\mathbf{z}, w) \in D \cap \Omega_{\varepsilon}$ and $k \in \mathbf{N}$;

by analytic continuation, the same equality holds for every $(\mathbf{z}, w)$ in the connected component $\Omega_{\varepsilon}^{\prime}$ of $\Omega_{\varepsilon}$ containing $\Omega \cap\{w=0\}$, 
therefore the series $\sum_{k=0}^{\infty} a_{k}(z) w^{k}$ converges to $f(\mathbf{z}, w)$ uniformly on compact sets of $\Omega_{\varepsilon}^{\prime}$.

To show that $\bigcup_{\varepsilon>0} \Omega_{\varepsilon}^{\prime}=\Omega$, fix $(\mathbf{z}, w) \in \Omega$, join it with $\Omega \cap\{w=0\}$ by a broken line $L$; since $\delta$ is lower semicontinuous it attains a positive minimum $\varepsilon_{0}$ on $L$, therefore $L \subseteq \Omega_{\varepsilon_{0} / 2}$ and $(\mathbf{z}, w) \in \Omega_{\varepsilon_{0} / 2}^{\prime}$.

Thus $(\pi(\Omega) \times \mathbf{C} ; \Omega)$ has the Runge property. So if $E(\pi(\Omega))$ is schlicht, then $E(\Omega)$ is schlicht by Lemma 1.1 and complete by Proposition 2.1. The remainder of the proof is now similar to that of Theorem 2.4 .

3. Circular domains. A domain $\Omega$ in $\mathbf{C}^{n}$ is a circular domain if $\mathbf{x}=\left(x_{1}, \ldots, x_{n}\right) \in \Omega$ implies $e^{i \theta} \mathbf{x} \in \Omega$ for any $\theta \in \mathbf{R}$. If $\Omega$ is a circular domain, set

$$
V_{\Omega}=\bigcup_{\lambda>0} \lambda \Omega
$$

note that $V_{\Omega}=\mathbf{C}^{n}$ if and only if $\mathbf{0} \in \Omega$. A complex cone (it need not be convex) will be a domain $\Omega$ with $V_{\Omega}=\Omega$. Let $s, t: V_{\Omega} \rightarrow[0,+\infty$ [ be defined by

$$
s(\mathbf{x})=\inf _{\alpha \mathbf{x} \in \Omega}|\alpha|, \quad t(\mathbf{x})=\inf _{\alpha \mathbf{x} \in \Omega}|\alpha|^{-1} ;
$$

thus $s, t$ are complex homogeneous of degree $-1,1$ respectively (i.e., $s(\alpha \mathbf{x})=|\alpha|^{-1} s(\mathbf{x}), t(\alpha \mathbf{x})=|\alpha| t(\mathbf{x})$ for each $\left.\alpha \in \mathbf{C}^{*}\right)$ and upper semicontinuous. A circular domain $\Omega$ will be said to have connected linear sections if for each $\mathbf{x} \in V_{\Omega}$ the set

$$
B_{\mathbf{x}}=\{\alpha \in \mathbf{C}: \alpha \mathbf{x} \in \Omega\}
$$

is connected. In this case $\Omega=\left\{\mathbf{x} \in V_{\Omega}: s(\mathbf{x})<1, \quad t(\mathbf{x})<1\right\}$.

As in the Hartogs case, note that if $\Omega$ is a circular domain with schlicht envelope of holomorphy then $E(\Omega)$ is itself circular.

Let $\Omega^{\prime}$ be a Hartogs domain not intersecting $\{w=0\}$. The map $h: \mathbf{C}^{n} \backslash\{w=0\} \rightarrow \mathbf{C}^{n} \backslash\left\{x_{n}=0\right\}$ given by $h(\mathbf{z}, w)=(w \mathbf{z}, w)$ is biholomorphic onto, and $\Omega=h\left(\Omega^{\prime}\right)$ is a circular domain (not intersecting $\left.\left\{x_{n}=0\right\}\right)$. Conversely, given a circular domain $\Omega$ which does not meet a hyperplane $\Sigma$ through 0 , we can change coordinates in $\mathbf{C}^{n}$ so that $\Sigma=\left\{x_{n}=0\right\}$; thus $h^{-1}(\Omega)$ is a Hartogs domain in $\mathbf{C}^{n} \backslash\{w=0\}$. Notice that in the above hypotheses $h\left(\pi\left(\mathbf{\Omega}^{\prime}\right) \times \mathbf{C}^{*}\right)=V_{\Omega}$. In view of this remark and of Lemma 1.2 , we will be able to transfer to the circular case most of the results and examples obtained in the preceding section. 
LEMMA 3.1. Let $\Omega$ be a circular domain with connected linear sections. Then each $f \in \mathscr{O}(\Omega)$ can be uniquely expanded into a series $\sum_{k \in \mathbf{Z}} r_{k}$ which converges uniformly on compact sets of $\Omega$, where $r_{k} \in \mathscr{O}\left(V_{\Omega}\right)$ is homogeneous of degree $k$, for $k \in \mathbf{Z}$; if $\mathbf{0} \in \mathbf{\Omega}$, then $r_{k}$ vanishes identically for all $k<0$, and is a homogeneous polynomial otherwise. Thus $\left(V_{\Omega} ; \Omega\right)$ has the Runge property.

Proof. For $k \in \mathbf{Z}$ define $r_{k}: V_{\Omega} \rightarrow \mathbf{C}$ by

$$
r_{k}(\mathbf{x})=\frac{1}{2 \pi i} \int_{|\alpha|=c} \frac{f(\alpha \mathbf{x})}{\alpha^{k+1}} d \alpha,
$$

where $c \in] s(\mathbf{x}), 1 / t(\mathbf{x})\left[\right.$ : by the Cauchy formula, $r_{k}(\mathbf{x})$ does not depend on the choice of $c$. So the function $r_{k}$ is homogeneous of degree $k$ and holomorphic. A standard computation shows that $\sum_{k} r_{k}$ converges uniformly to $f$ on compact sets of $\Omega$. The uniqueness follows from that of the Laurent expansion. If $\mathbf{0} \in \Omega$, it is evident that $r_{k}$ equals the homogeneous component of degree $k$ of the Taylor expansion of $f$ at $\mathbf{0}$ for $k \geq 0$, and vanishes identically for $k<0$.

Of course, to ensure that $r_{k} \equiv 0$ for negative $k$, it is enough that $E(\Omega)$ is schlicht and contains 0 .

We now need a special case of forthcoming Proposition 3.7 and Theorem 3.8.

LEMMA 3.2. If $\Omega$ is a circular domain with connected linear sections such that $V_{\Omega}$ equals $\mathbf{C}^{n}$ or $\mathbf{C}^{n} \backslash\{\mathbf{0}\}$, then $E(\Omega)$ is schlicht and contains 0.

Proof. By Lemma 1.1 and Lemma 3.1, $E(\Omega)$ is schlicht and $\left(\mathbf{C}^{n} ; E(\Omega)\right)$ is Runge. Take a line $r$ through $\mathbf{0}$, and $g \in \mathscr{O}(E(\Omega) \cap r)$. Since $E(\Omega)$ is a domain of holomorphy, by Cartan's theorem B there exists $\tilde{g} \in \mathscr{O}(E(\Omega))$ which extends $g$. Therefore $(r ; E(\Omega) \cap r)$ is Runge; by the Runge theorem $E(\Omega) \cap r$ is simply connected, so $\mathbf{0} \in E(\boldsymbol{\Omega})$.

The assumption of connected linear sections in Lemma 3.2 cannot be dropped, as we show with

EXAMPLE 3.3. A circular domain $\Omega$ in $\mathbf{C}^{2}$ such that $V_{\Omega}=\mathbf{C}^{2} \backslash\{\boldsymbol{0}\}$ but $E(\Omega)$ is not schlicht. 
Define a Riemann domain $R=(R, \tau)$ on $\widehat{\mathbf{C}}=\mathbf{C} \cup\{\infty\}$ through three charts $S, L_{1}, L_{2} \subset R$, as follows. Set

$$
\begin{aligned}
\tau(S) & =\widehat{\mathbf{C}} \backslash\{\lambda \in \mathbf{C}:|| \lambda|-1| \leq 1 / 2, \operatorname{Re} \lambda \geq 0, \operatorname{Im} \lambda \geq 0\}, \\
\tau\left(L_{1}\right) & =\left\{\lambda \in \mathbf{C}^{*}:|| \lambda|-1|<1 / 4,-\pi / 2<\arg \lambda<3 \pi / 2\right\}, \\
\tau\left(L_{2}\right) & =\left\{\lambda \in \mathbf{C}^{*}:|| \lambda|-1|<3 / 4, \pi<\arg \lambda<3 \pi\right\},
\end{aligned}
$$

where

$$
\begin{aligned}
\tau\left(S \cap L_{1}\right) & =\left\{\lambda \in \tau(S) \cap \tau\left(L_{1}\right): \operatorname{Re} \lambda>0\right\}, \\
\tau\left(L_{1} \cap L_{2}\right) & =\left\{\lambda \in \tau\left(L_{1}\right) \cap \tau\left(L_{2}\right): \operatorname{Re} \lambda<0, \operatorname{Im} \lambda<0\right\}, \\
\tau\left(S \cap L_{2}\right) & =\varnothing .
\end{aligned}
$$

Note that $\tau: R \rightarrow \widehat{\mathbf{C}}$ is onto.

Define further $r: R \rightarrow[0,1 / 2[$ by

$$
r(\xi)= \begin{cases}0 & \text { if } \xi \in S, \\ \frac{\arg \tau(\xi)}{6 \pi} & \text { if } \xi \in R \backslash S,\end{cases}
$$

the choice of the argument being the one appearing in the definitions of $L_{1}$ and $L_{2}$; note that $r$ is continuous. Let $q: \mathbf{C}^{2} \backslash\{\boldsymbol{0}\} \rightarrow \widehat{\mathbf{C}}$ be given by $q(\mathbf{x})=x_{1} / x_{2}$, and

$\Omega=\left\{\mathbf{x} \in \mathbf{C}^{2} \backslash\{\mathbf{0}\}:\right.$ there exists $\xi \in R$ such that $\tau(\xi)=q(\mathbf{x})$, and $|\|\mathbf{x}\|-1-r(\xi)|<1 / 25$ or $|\|\mathbf{x}\|-1+r(\xi)|<1 / 25\}$;

observe that, for each $\mathbf{x} \in \Omega$, the above $\xi$ is unique; thus a continuous and holomorphic map $\theta: \Omega \rightarrow R$ is defined which satisfies $\tau \circ \theta=q$.

If

$D=\left\{\mathbf{x} \in\left(\mathbf{C}^{*}\right)^{2}:|| q(\mathbf{x})|-1|<1 / 4, \quad|\|\mathbf{x}\|-1|<\frac{\arg q(\mathbf{x})}{6 \pi}<1 / 4\right\}$, given $f \in \mathscr{O}(\Omega)$, define $\tilde{f}: D \rightarrow \mathbf{C}$ by:

$$
\tilde{f}(\mathbf{x})=\frac{1}{2 \pi i}\left[\int_{|\alpha|=c_{+}}-\int_{|\alpha|=c_{-}}\right] \frac{f(\alpha \mathbf{x})}{\alpha-1} d \alpha
$$

where

$$
c_{ \pm}=\left[1 \pm \frac{\arg q(\mathbf{x})}{6 \pi}\right] /\|\mathbf{x}\| .
$$

Since $\Omega$ is open, by Cauchy's theorem we can locally replace with a constant the radius of the circle on which $\alpha$ ranges, without changing 
$\tilde{f}$; thus, by Cauchy's formula, $\tilde{f}$ is holomorphic and agrees with $f$ on $\{\mathbf{x} \in D: \arg q(\mathbf{x})<6 \pi / 25\}$.

Because $R$ is simply connected and noncompact, Riemann's uniformization theorem furnishes a one-to-one holomorphic function $F: R \rightarrow \mathbf{C}$. So $f=F \circ \theta: \Omega \rightarrow \mathbf{C}$ is holomorphic. The points $\mathbf{y}=$ $(-1 / \sqrt{2}, 1 / \sqrt{2}), \mathbf{z}=13 \mathbf{y} / 15$ belong to $\Omega \cap D$. Since $\theta(\mathbf{y}) \in S$, $\theta(\mathbf{z}) \in L_{1}$, with $\theta(\mathbf{y}) \neq \theta(\mathbf{z})$, we have that $f(\mathbf{y}) \neq f(\mathbf{z})$. On the other hand, the image through $q$ of the whole segment (contained in $D$ ) joining $\mathbf{y}$ to $\mathbf{z}$ is $\{-1\}$. However, the identity $0 \equiv$ $\left.(\partial / \partial \alpha) f(\alpha \mathbf{x})\right|_{\alpha=1} \equiv x_{1}\left(\partial f / \partial x_{1}\right)(\mathbf{x})+x_{2}\left(\partial f / \partial x_{2}\right)(\mathbf{x})$ still holds for $\tilde{f}$ on $D$ by analytic continuation; it follows that $\tilde{f}(\mathbf{y})=\tilde{f}(\mathbf{z})$. Therefore $E(\Omega)$ is not schlicht.

Proposition 3.4. Let $\Omega$ be a circular domain with connected linear sections. Then $\Omega$ is a domain of holomorphy if and only if the following conditions are both fulfilled:

(1) $V_{\Omega}$ is a domain of holomorphy;

(2) $s, t$ are plurisubharmonic.

Condition (2) can be replaced by:

$\left(2^{\prime}\right) \log s, \log t$ are plurisubharmonic.

Proof. The "if" part follows from the equality $\Omega=\left\{\mathbf{x} \in V_{\Omega}: s(\mathbf{x})<\right.$ $1, t(\mathbf{x})<1\}$.

Suppose $\Omega$ is a domain of holomorphy. Using the map $h$ and invoking Proposition 2.1 we prove that $V_{\Omega} \backslash \Sigma$ is a domain of holomorphy for every hyperplane $\Sigma$ through 0 . Lemma 1.2 guarantees that $E\left(V_{\Omega}\right)$ is schlicht and $V_{\Omega} \backslash \Sigma=E\left(V_{\Omega}\right) \backslash \Sigma$ for every $\Sigma$. Thus $V_{\Omega} \backslash\{\boldsymbol{0}\}=$ $E\left(V_{\Omega}\right) \backslash\{0\}$. Now, $E\left(V_{\Omega}\right)$ is a complex cone, so if $\mathbf{0} \in E\left(V_{\Omega}\right)$ then $E\left(V_{\Omega}\right)=\mathbf{C}^{n}$ and $\mathbf{C}^{n} \backslash\{\boldsymbol{0}\} \subset V_{\Omega}$, so, by Lemma 3.2, we have $V_{\Omega}=\mathbf{C}^{n}$.

If $a, b$ are associated to the Hartogs domain $h^{-1}\left(\Omega \backslash\left\{x_{n}=0\right\}\right)$, then the complex homogeneity of $s, t$ gives

$$
\begin{aligned}
\log s(\mathbf{x}) & =\log s\left(\mathbf{x} / x_{n}\right)-\log \left|x_{n}\right| \\
& =\log a\left(x_{1} / x_{n}, \ldots, x_{n-1} / x_{n}\right)-\log \left|x_{n}\right|, \\
\log t(\mathbf{x}) & =-\log b\left(x_{1} / x_{n}, \ldots, x_{n-1} / x_{n}\right)+\log \left|x_{n}\right| .
\end{aligned}
$$

Using Proposition 2.1 again and changing coordinates we prove that $\log s, \log t$ are plurisubharmonic on $V_{\Omega} \backslash\{\boldsymbol{0}\}$. If $V_{\Omega}=\mathbf{C}^{n}$, then $\mathbf{0} \in \Omega$, so $\log s(\mathbf{0})=\log t(\mathbf{0})=-\infty$. In any case, $\log s, \log t$ are plurisubharmonic on $V_{\Omega}$.

It is well known that $\left(2^{\prime}\right)$ implies (2). 
Here is an interesting consequence of Proposition 3.4:

COROLlaRY 3.5. Let $V$ be a complex cone, and $u: V \rightarrow[0,+\infty[$ be an upper semicontinuous function, complex homogeneous of degree $\gamma \neq 0$. Then $u_{*}=u_{(*)}$. In fact $u$ is plurisubharmonic if and only if $\log u$ is; provided $V$ is a domain of holomorphy, this is also equivalent to saying that $\Omega=\{\mathbf{x} \in V: u(\mathbf{x})<1\}$ is a domain of holomorphy.

Proof. Observe first that $\Omega$ is connected because $V$ is. Suppose $V$ is a domain of holomorphy. If $\gamma>0$, then $u^{1 / \gamma}$ is the $t$ function of $\Omega$. If, in addition, $u$ is plurisubharmonic then $\Omega$ is a domain of holomorphy, and by Proposition 3.4 the function $\log u=\gamma \log t$ is plurisubharmonic. Instead, if $\gamma<0$ then $u^{-1 / \gamma}$ is the $s$ function of $\Omega$, and we conclude as before.

If $V$ is not a domain of holomorphy (so $0 \notin V$ ), given $\mathbf{x}^{0} \in V$ we can assume $\mathbf{x}_{n}^{0} \neq 0$. Now

$$
\left\{\mathbf{x} \in \mathbf{C}^{n}: x_{n} \neq 0, \max _{1 \leq j \leq n-1}\left|x_{j} / x_{n}-x_{j}^{0} / x_{n}^{0}\right|<\delta\right\}
$$

is a complex cone and a domain of holomorphy containing $\mathbf{x}^{0}$, which is contained in $V$ for small $\delta>0$. With this argument we reduce to the previous case.

The equality $u_{*}=u_{(*)}$ now follows trivially.

The domain $h(\Omega)$, where $\Omega$ is given in Example 2.2, is a circular domain of holomorphy which is not with connected linear sections and is such that $V_{h(\Omega)}$ is not a domain of holomorphy. Furthermore $\log s, \log t$ are not plurisubharmonic; by Corollary 3.5, neither of $s, t$ is plurisubharmonic.

The assumption of connected linear sections is essential in Proposition 3.4 also.

EXAMPLE 3.6. A circular domain of holomorphy $\Omega$ in $\mathbf{C}^{2}$ which is not with connected linear sections and is such that $V_{\Omega}=\mathbf{C}^{2} \backslash\{\mathbf{0}\}$.

Let $\sigma: \mathbf{C}^{2} \rightarrow[0,+\infty[$ be given by

$$
\sigma(\mathbf{x})=\left(\|\mathbf{x}\|^{2}-1\right)^{2} / 2+2\left(\operatorname{Im} x_{1} \overline{x_{2}}\right)^{2},
$$

and set

$$
\Sigma_{\delta}= \begin{cases}\{\sigma<\delta\} & \text { for } \delta>0 \\ \{\sigma=0\} & \text { for } \delta=0 .\end{cases}
$$

Therefore $\Sigma_{0}$ is the compact set $\left\{e^{i \theta}(\cos \phi, \sin \phi): \theta, \phi \in \mathbf{R}\right\}$, and the complex hessian $\left(\partial^{2} \sigma / \partial x_{j} \partial \overline{x_{k}}\right)_{j, k=1,2}$ of $\sigma$ equals the identity matrix at every point of it, therefore $\sigma$ is strictly plurisubharmonic there. 
Let $\rho:[-1,1] \rightarrow[0,1]$ be a smooth monotonic function such that $\{\rho=0\}=[-1,-1 / 2]$ and $\{\rho=1\}=[1 / 2,1]$; let $q: \mathbf{C}^{2} \backslash\{\mathbf{0}\} \rightarrow$ $[0,1]$ be given by

$$
q(\mathbf{x})=\rho\left(\frac{\left|x_{2}\right|^{2}-\left|x_{1}\right|^{2}}{\|\mathbf{x}\|^{2}}\right) \quad \text { for each } \mathbf{x} \in \mathbf{C}^{2} \backslash\{\mathbf{0}\} .
$$

Using the identities

$$
\begin{aligned}
\left(\operatorname{Im} x_{1} \overline{x_{2}}\right)^{2} & =\left|x_{1}\right|^{2}\left|x_{2}\right|^{2}-\left(\operatorname{Re} x_{1} \overline{x_{2}}\right)^{2}, \\
\left.|| x_{2}\right|^{2}-\left|x_{1}\right|^{2} \mid & =\left(\|\left.\mathbf{x}\right|^{4}-4\left|x_{1}\right|^{2}\left|x_{2}\right|^{2}\right)^{1 / 2},
\end{aligned}
$$

it is easy to see that $\Sigma_{1 / 10} \cap\left\{\operatorname{Re} x_{1} \overline{x_{2}}=0\right\}$ is relatively compact in $\{q=0$ or $=1\}=\left\{\left|x_{1} / x_{2}\right| \geq \sqrt{3}\right.$ or $\left.\leq 1 / \sqrt{3}\right\}$.

Choose $\alpha>0$ small enough that $\sigma \circ \eta_{+}$is still strictly plurisubharmonic on $\eta_{+}^{-1}\left(\Sigma_{0}\right)$, where $\eta_{+}(\mathbf{x})=e^{\alpha q(\mathbf{x})} \mathbf{x}$. Then choose $\beta \in$ ]0,1/10[ small enough that $\sigma \circ \eta_{+}$is strictly plurisubharmonic on $\eta_{+}^{-1}\left(\Sigma_{\beta}\right)$, and that $L_{+}\left(\Sigma_{\beta}\right)$ is disjoint from $\Sigma_{\beta}$, where $L_{+}(\mathbf{x})=e^{\alpha} \mathbf{x}$. Finally, choose $\gamma>0$ small enough that $\sigma \circ \eta_{-}$is strictly plurisubharmonic on $\eta_{-}^{-1}\left(\Sigma_{\beta}\right)$, where $\eta_{-}(\mathbf{x})=\left(e^{i \gamma q(\mathbf{x})} x_{1}, x_{2}\right)$, and that $V_{\bigcup_{k=0}^{N} L_{-}^{k}\left(\Sigma_{\beta / 2}\right)}=\mathbf{C}^{2} \backslash\{\boldsymbol{0}\}$ for some $N \in \mathbf{N}$, where $L_{-}(\mathbf{x})=$ $\left(e^{-i \gamma} x_{1}, x_{2}\right)$.

Let $L=L_{-} \circ L_{+}$, and

$$
\begin{aligned}
A_{ \pm} & =\eta_{ \pm}^{-1}\left(\Sigma_{\beta / 2} \cap\left\{ \pm \operatorname{Re} x_{1} \overline{x_{2}}>0, \text { or }\left|x_{1}\right|>2\left|x_{2}\right| \text { or }\left|x_{2}\right|>2\left|x_{1}\right|\right\}\right), \\
B_{2 k} & =L\left(A_{+}\right), \quad B_{2 k+1}=L\left(A_{-}\right) \text {for } k=0, \ldots, N, \\
\Omega & =\bigcup_{j=0}^{2 N+1} B_{j} .
\end{aligned}
$$

The domains $A_{ \pm}$are thus intersection of pseudoconvex domains at each of their boundary points (in fact $\left\{\left|x_{1}\right|>2\left|x_{2}\right|\right\},\left\{\left|x_{2}\right|>2\left|x_{1}\right|\right\}$ are domains of holomorphy), so the same holds for $B_{j}$ for any $j=$ $0, \ldots, 2 N+1$. Moreover $B_{j} \cap B_{j^{\prime}}$ is empty for $\left|j-j^{\prime}\right| \geq 2$, and:

$$
\begin{gathered}
B_{2 k} \cap B_{2 k+1}=L^{k}\left(\Sigma_{\beta / 2}\right) \cap\left\{\left|x_{1}\right|>2\left|x_{2}\right|\right\} \\
\quad \text { is relatively compact in }\{q=0\}, \text { and } \\
\left(B_{2 k} \cup B_{2 k+1}\right) \cap\{q=0\}=L^{k}\left(\Sigma_{\beta / 2}\right) \cap\{q=0\} \\
\quad \text { is a domain of holomorphy; while } \\
B_{2 k+1} \cap B_{2 k+2}=L^{k}\left(L_{-}\left(\Sigma_{\beta / 2}\right)\right) \cap\left\{\left|x_{2}\right|>2\left|x_{1}\right|\right\} \\
\text { is relatively compact in }\{q=1\}, \text { and } \\
\left(B_{2 k+1} \cup B_{2 k+2}\right) \cap\{q=1\}=L^{k}\left(L_{-}\left(\Sigma_{\beta / 2}\right)\right) \cap\{q=1\}
\end{gathered}
$$

is a domain of holomorphy. 
Therefore $\Omega$ is a circular domain of holomorphy, is not with connected linear sections, and $V_{\Omega}=\mathbf{C}^{2} \backslash\{\boldsymbol{0}\}$. Notice finally that the $s, t$ functions of $\Omega$ are not plurisubharmonic.

If the circular domain $\Omega$ contains $\mathbf{0}$, the hypothesis of connected linear sections is not necessary, and from [Ct, Théorème I p. 14] (see also [BM, Theorem IV.10 p. 79]) together with [B, Theorem I.(c) p. 527] the following can be derived:

Proposition 3.7. If $\Omega \subset \mathbf{C}^{n}$ is a circular domain which contains $\mathbf{0}$, then $E(\Omega)$ is schlicht and

$$
E(\Omega)=\left\{\mathbf{x} \in \mathbf{C}^{n}: t_{*}(\mathbf{x})<1\right\} .
$$

Hence $\left(\mathbf{C}^{n} ; E(\Omega)\right)$ is Runge.

As to circular domains not containing $\mathbf{0}$, the main result is the following:

THeOREM 3.8. Let $\Omega$ be a circular domain with connected linear sections such that $\mathbf{0} \notin \Omega$. Then $E(\Omega)$ is schlicht and with connected linear sections if and only if $E\left(V_{\Omega}\right)$ is schlicht. In this case $E\left(V_{\Omega}\right)=$ $V_{E(\Omega)}$, the pair $\left(E\left(V_{\Omega}\right) ; E(\Omega)\right)$ is Runge, and

$$
E(\Omega)=\left\{\mathbf{x} \in E\left(V_{\Omega}\right): s_{*}(\mathbf{x})<1, t_{*}(\mathbf{x})<1\right\} .
$$

Proof. As done for Proposition 3.4, the equivalence in the statement can be easily derived from Theorem 2.4 by taking away hyperplanes through zero from the domain in consideration, invoking Lemma 1.2 and using the map $h$; same for the identity $E\left(V_{\Omega}\right)=V_{E(\Omega)}$. Lemma 3.1 now yields that $\left(E\left(V_{\Omega}\right) ; E(\Omega)\right)$ is Runge. The description of $E(\Omega)$ in terms of $s_{*}, t_{*}$ follows from Proposition 3.4 exactly as the corresponding description in Theorem 2.4 follows from Proposition 2.1.

Note that $h(D)$, where $D$ is the domain of Remark 2.6, has connected linear sections, but its schlicht envelope of holomorphy does not.

4. Interpretation in the projective space. We shall say that a domain $D \subset \mathbf{P}^{n-1}(\mathbf{C})$ has schlicht envelope of holomorphy $E(D) \subset \mathbf{P}^{n-1}(\mathbf{C})$ if the domain $E(D)$ contains $D$, is Stein, and every holomorphic function on $D$ extends to $E(D)$ holomorphically.

Let $p: \mathbf{C}^{n} \backslash\{\boldsymbol{0}\} \rightarrow \mathbf{P}^{n-1}(\mathbf{C})$ be the canonical projection. If $\boldsymbol{\Omega} \subset \mathbf{C}^{n}$ is a circular domain not containing $\mathbf{0}$, then $p(\Omega)$ is a domain in $\mathbf{P}^{n-1}(\mathbf{C})$, and $V_{\Omega}=p^{-1}(p(\Omega))$. 
THEOREM 4.1. Let $V$ be a complex cone not containing 0 . Then:

(1) $V$ is a domain of holomorphy if and only if $p(V)$ is a Stein domain in $\mathbf{P}^{n-1}(\mathbf{C})$;

(2) $E(V)$ is schlicht and $\neq \mathbf{C}^{n}$ if and only if $E(p(V))$ is schlicht. In this case $E(V)=p^{-1}(E(p(V)))$;

(3) $E(V)=\mathbf{C}^{n}$ if and only if $\mathscr{O}(p(V))=\mathbf{C}$.

Proof. (1) Suppose that $V$ is Stein. Chosen a sequence $\left(\mathbf{x}_{\nu}\right)_{\nu \in \mathbf{N}}$ of isolated points in $p(V)$, and a sequence $\left(\alpha_{\nu}\right)_{\nu \in \mathbf{N}}$ of complex numbers, we must prove that there exists a holomorphic function $f$ on $p(V)$ such that $f\left(\mathbf{x}_{\nu}\right)=\alpha_{\nu}$ for every $\nu \in \mathbf{N}$. The analytic set $I=\bigcup_{\nu \in \mathbf{N}} p^{-1}\left(\mathbf{x}_{\nu}\right)$ is closed in $V$, and the function $\phi: I \rightarrow \mathbf{C}$ such that $\left.\phi\right|_{p^{-1}\left(\mathbf{x}_{\nu}\right)} \equiv \alpha_{\nu}$ is holomorphic on $I$, therefore, by Cartan's theorem B, there exists a holomorphic function $\phi^{\prime}$ on $V$ such that $\left.\phi^{\prime}\right|_{I} \equiv \phi$. Let $\sum_{k \in \mathbf{Z}} r_{k}$ be the series expansion of $\phi^{\prime}$ as in Lemma 3.1. We obtain that $\left.r_{0}\right|_{p^{-1}\left(\mathbf{x}_{\nu}\right)} \equiv \alpha_{\nu}$ for every $\nu \in \mathbf{N}$; therefore $r_{0}$ projects to a holomorphic function $f$ on $p(V)$ with the required properties.

Conversely, suppose that $p(V)$ is Stein. For every hyperplane $\Sigma$ through $\mathbf{0}$ in $\mathbf{C}^{n}$, both $\mathbf{C}^{n} \backslash \Sigma$ and $p\left(\mathbf{C}^{n} \backslash \Sigma\right)=\mathbf{P}^{n-1}(\mathbf{C}) \backslash p(\Sigma)$ are Stein domains, so $V \backslash \Sigma=p^{-1}(p(V) \backslash p(\Sigma))$ is Stein. If $V$ were $\mathbf{C}^{n} \backslash\{\boldsymbol{0}\}$, then $p(V)$ would be $p\left(\mathbf{C}^{n} \backslash\{\mathbf{0}\}\right)=\mathbf{P}^{n-1}(\mathbf{C})$, which is not Stein. As in Proposition 3.4, the only possibility left is that $V$ be a domain of holomorphy in $\mathbf{C}^{n}$.

(2) Assume $E(V)$ is schlicht and different from $\mathbf{C}^{n}$. Then every holomorphic function, homogeneous of degree 0 , defined on $V$ can be extended to a holomorphic homogeneous function on $E(V) \subset$ $\mathbf{C}^{n} \backslash\{\boldsymbol{0}\}$. Thus every holomorphic function on $p(V)$ can be extended to $p(E(V))$, which is Stein by (1): so $E(p(V))=p(E(V))$; moreover, since $E(V)$ is a cone, $E(V)=p^{-1}(E(p(V)))$.

Suppose now that $p(V)$ has a schlicht envelope of holomorphy $E(p(V))$. If $H$ is the hyperplane bundle on $\mathbf{P}^{n-1}(\mathbf{C})$ (see [SS]), by $\bigotimes^{k} H$ we will denote its $k$ th tensor power for each $k \in \mathbf{Z}$ : for $k<0$ we set $\bigotimes^{k} H=\bigotimes^{-k} H^{*}$. The homogeneous holomorphic functions of degree $k$ on $V$ can be identified with holomorphic sections of $\left.\bigotimes^{k} H\right|_{p(V)}$. Since $E(p(V))$ is Stein, then $F_{k}=\left.\otimes^{k} H\right|_{E(p(V))}$ is also Stein, so it can be embedded as a closed analytic subset of $\mathbf{C}^{N}$, for $N$ sufficiently large. Thus every holomorphic map $\psi: p(V) \rightarrow F_{k}$ can be extended to a holomorphic map $\tilde{\psi}: E(p(V)) \rightarrow F_{k}$; furthermore, by analytic continuation, if $\psi$ is a section of $\left.F_{k}\right|_{p(V)}$, then $\tilde{\psi}$ is also a 
section of $F_{k}$. We have thus proved that every holomorphic function on $V$, homogeneous of degree $k$, can be extended to a holomorphic function, homogeneous of the same degree, on $V^{\prime}=p^{-1}(E(p(V)))$. Now, as follows from (1), Lemma 3.1, and Lemma 1.1, $E(V)$ must be schlicht and contained in $V^{\prime}$ (so $E(V) \neq \mathbf{C}^{n}$ ).

(3) If $E(V)=\mathbf{C}^{n}$ then obviously $\mathscr{O}(p(V))=\mathbf{C}$. Conversely assume that $\mathscr{O}(p(V))=\mathrm{C}$ and $\phi$ is a holomorphic homogeneous function on $V$ of degree $k<0$ : then $\phi(\mathbf{x}) x_{1}^{-k}, \phi(\mathbf{x}) x_{2}^{-k}$ are constant. If $\phi$ did not vanish identically then $\left(\phi(\mathbf{x}) x_{1}^{-k}\right) /\left(\phi(\mathbf{x}) x_{2}^{-k}\right)=\left(x_{1} / x_{2}\right)^{-k}$ would be constant on a nonempty open subset of $\mathbf{C}^{n}$, but this is absurd: so $\phi \equiv 0$. We now want to prove by induction that if $\phi \in \mathscr{O}(V)$ is homogeneous of degree $k \geq 0$ then it can be extended to $\mathbf{C}^{n}$. If $k=0$, this is the hypothesis. If $k>0$, then the functions $\psi_{j}=$ $\partial \phi / \partial x_{j}$ are homogeneous of degree $k-1$, so they have a holomorphic and homogeneous extension $\tilde{\psi}_{j}$ to $\mathbf{C}^{n}$ by the inductive hypothesis. The differential form $\sum_{j=1}^{n} \tilde{\psi}_{j} d x_{j}$ is $\partial$-closed on $\mathbf{C}^{n}$, so there exists a function $\tilde{\phi}$ holomorphic on $\mathbf{C}^{n}$ such that $\left.\left(\partial \tilde{\phi} / \partial x_{j}\right)\right|_{V}=\partial \phi / \partial x_{j}$ for each $j$. Therefore $\tilde{\phi}$ can be chosen so that $\left.\tilde{\phi}\right|_{V}=\phi$. By analytic continuation $\tilde{\phi}$ is homogeneous of degree $k$. Again by Lemma 1.1 and Lemma 3.1 we obtain that $E(V)$ is schlicht. If $E(V)$ were different from $\mathbf{C}^{n}$, then we would have $p(V) \subset p(E(V))$, which is Stein: this is absurd because $\mathscr{O}(p(V))=\mathbf{C}$.

REMARK 4.2. Let $\alpha: \mathbf{C}^{n} \backslash\{\mathbf{0}\} \rightarrow \mathbf{P}^{n-1}(\mathbf{C}) \times\left(\mathbf{C}^{n} \backslash\{\mathbf{0}\}\right)$ be given by $\alpha(\mathbf{x})=(p(\mathbf{x}), \mathbf{x})$. Then for each cone $V$ not containing $\mathbf{0}$, the map $\left.\alpha\right|_{V}$ is a biholomorphism of $V$ onto the total space $K_{p(V)}$ of the restriction of the tautological line bundle to $p(V)$ (in the language of Theorem 4.1, such bundle is $\left.\left.H^{-1}\right|_{p(V)}\right)$. Let now $\Omega \subset \mathbf{C}^{n}$ be a circular domain with connected linear sections and not containing $\mathbf{0}$. Assume that the corresponding $s, t$ functions are $\mathscr{C}^{\infty}$ and never vanish: then $s, 1 / t$ naturally give rise to hermitian metrics $h_{s}, h_{1 / t}$ on $\left.H^{-1}\right|_{p\left(V_{\Omega}\right)}$, and $\Omega$ is the inverse image through $\alpha$ of

$$
\left\{\mathbf{x} \in K_{p\left(V_{\Omega}\right)}:\|\mathbf{x}\|_{h_{s}}<1,\|\mathbf{x}\|_{h_{1 / t}}>1\right\} .
$$

Proposition 3.4, for instance, can be restated in this case by saying that $\Omega$ is a domain of holomorphy if and only if $p(\Omega)$ is Stein and the curvature forms of $h_{s}, h_{1 / t}$ are semipositive, seminegative respectively (see [SS]). A similar statement holds also if one of $s, t$ vanishes identically, whereas the other is $\mathscr{C}^{\infty}$ and never vanishes. 
Corollary 4.3. If $\Omega$ is a circular domain with connected linear sections, then:

(1) $E(\Omega)$ is schlicht and contains $\mathbf{0}$ if and only if $\mathscr{O}(p(\Omega \backslash\{\mathbf{0}\}))=\mathbf{C}$;

(2) $E(\Omega)$ is schlicht, does not contain $\mathbf{0}$ and has connected linear sections if and only if $\Omega$ does not contain $\mathbf{0}$ and $E(p(\Omega))$ is schlicht. In this case $E(p(\Omega))=p(E(\Omega))$.

Proof. Follows from Theorem 3.8 and Theorem 4.1 (in the "only if" part of (1), $E(\Omega)$ has connected linear sections by Proposition 3.7).

COROLlaRy 4.4. If $\Omega \subset \mathbf{C}^{2}$ is a circular domain with connected linear sections, then $E(\Omega)$ is schlicht and has connected linear sections, and $V_{E(\Omega)}$ equals $V_{\Omega}$ or $V_{\Omega} \cup\{\boldsymbol{0}\}$.

Proof. If $\mathbf{0} \in \Omega$ then the thesis follows from Proposition 3.7; whereas if $\mathbf{0} \notin \Omega$, then $p(\Omega)$ is either Stein or the whole $\mathbf{P}^{1}(\mathbf{C})$. We conclude using Theorem 3.8 and Theorem 4.1.

\section{REFERENCES}

[B] Th. J. Barth, The Kobayashi indicatrix at the center of a circular domain, Proc. Amer. Math. Soc., 88 (1983), 527-530.

[BM] S. Bochner and W. T. Martin, Several complex variables, Princeton Math. Ser. \#10, Princeton University Press, Princeton, 1948.

[Cm] R. Carmignani, Envelopes of holomorphy and holomorphic convexity, Trans. Amer. Math. Soc., 179 (1973), 415-431.

[Ct] H. Cartan, Les fonctions de deux variables complexes et le problème de la représentation analytique, J. Math. Pures Appl., (9) 10 (1931), 1-114.

[GF] H. Grauert and K. Fritzsche, Several complex variables, Graduate Texts in Math. \#38, Springer, New York, 1976.

[GrR] H. Grauert and R. Remmert, Konvexität in der komplexen Analysis (Nichtholomorph-konvexe Holomorphiegebiete und Anwendungen auf die Abbildungstheorie), Comment. Math. Helv., 31 (1956/57), 152-183.

[GuR] R. C. Gunning and H. Rossi, Analytic functions of several complex variables, Ser. in Modern Anal., Prentice-Hall, Englewood Cliffs, 1965.

[H] L. Hörmander, An introduction to complex analysis in several variables, NorthHolland Math. Library \#7, North-Holland, Amsterdam, 1973.

[SS] B. Shiffman and A. J. Sommese, Vanishing theorems on complex manifolds, Progr. Math. \#56, Birkhäuser, Boston, 1985.

[T] P. Thullen, Zur Theorie der Singularitäten der Funktionen zweier komplexen Veränderlichen (Die Regularitätshüllen), Math. Ann., 106 (1932), 64-76.

[V] V. S. Vladimirov, Metody teorii funktsii mnogikh kompleksnykh peremennykh, Izdat. "Nauka", Moscow, 1964; English transl., Methods of the theory of functions of many complex variables, The M.I.T. Press, Cambridge, 1966. 
[VS] V. S. Vladimirov and M. Shirinbekov, $O$ postroenii obolochek golomorfnosti dlja oblastei Gartogsa, Ukrain. Mat. Zh., 15 (1963), 189-192; On the construction of envelopes of holomorphy for Hartogs domains, abstract \# 3175, MR 28 (1964), p. 620.

Received November 18, 1988 and in revised form February 8, 1990.

UNIVERSITȦ DI TRENTO

38050-Povo (TRENTo), ITALY

AND

UNIVERSITÀ DI ROMA "TOR VeRgATA"

Via FonTANILE DI CARCARICOLA

00133-Roma, Italy 



\section{PACIFIC JOURNAL OF MATHEMATICS EDITORS}

\author{
V. S. VARAdaraJan \\ (Managing Editor) \\ University of California \\ Los Angeles, CA 90024-1555-05 \\ Herbert Clemens \\ University of Utah \\ Salt Lake City, UT 84112 \\ THOMAS ENRIGHT \\ University of California, San Diego \\ La Jolla, CA 92093
}

R. FINN

Stanford University

Stanford, CA 94305

Hermann FlaschKa

University of Arizona

Tucson, AZ 85721

VAUGHAN F. R. Jones

University of California

Berkeley, CA 94720

STEVEN KerckHofF

Stanford University

Stanford, CA 94305

\section{C. MOORE}

University of California

Berkeley, CA 94720

Martin ScharlemanN

University of California

Santa Barbara, CA 93106

HAROLd STARK

University of California, San Diego

La Jolla, CA 92093
R. ARENS
E. F. BECKENBACH
F. WoLF
K. YoshidA
ASSOCIATE EDITORS

\begin{tabular}{ll}
\multicolumn{1}{c}{$(1906-1982)$} & \multicolumn{1}{c}{$(1904-1989)$} \\
SUPPORTING & INSTITUTIONS \\
UNIVERSITY OF ARIZONA & UNIVERSITY OF OREGON \\
UNIVERSITY OF BRITISH COLUMBIA & UNIVERSITY OF SOUTHERN CALIFORNIA \\
CALIFORNIA INSTITUTE OF TECHNOLOGY & STANFORD UNIVERSITY \\
UNIVERSITY OF CALIFORNIA & UNIVERSITY OF HAWAII \\
MONTANA STATE UNIVERSITY & UNIVERSITY OF TOKYO \\
UNIVERSITY OF NEVADA, RENO & UNIVERSITY OF UTAH \\
NEW MEXICO STATE UNIVERSITY & WASHINGTON STATE UNIVERSITY \\
OREGON STATE UNIVERSITY & UNIVERSITY OF WASHINGTON
\end{tabular}

The Supporting Institutions listed above contribute to the cost of publication of this Journal, but they are not owners or publishers and have no responsibility for its content or policies.

Mathematical papers intended for publication in the Pacific Journal of Mathematics should be in typed form or offset-reproduced (not dittoed), double spaced with large margins. Please do not use built up fractions in the text of the manuscript. However, you may use them in the displayed equations. Underline Greek letters in red, German in green, and script in blue. The first paragraph must be capable of being used separately as a synopsis of the entire paper. In particular it should contain no bibliographic references. Please propose a heading for the odd numbered pages of less than 35 characters. Manuscripts, in triplicate, may be sent to any one of the editors. Please classify according to the 1991 Mathematics Subject Classification scheme which can be found in the December index volumes of Mathematical Reviews. Supply name and address of author to whom proofs should be sent. All other communications should be addressed to the managing editor, or Elaine Barth, University of California, Los Angeles, California 90024-1555-05.

There are page-charges associated with articles appearing in the Pacific Journal of Mathematics. These charges are expected to be paid by the author's University, Government Agency or Company. If the author or authors do not have access to such Institutional support these charges are waived. Single authors will receive 50 free reprints; joint authors will receive a total of 100 free reprints. Additional copies may be obtained at cost in multiples of 50 .

The Pacific Journal of Mathematics (ISSN 0030-8730) is published monthly except for July and August. Regular subscription rate: $\$ 190.00$ a year (10 issues). Special rate: $\$ 95.00$ a year to individual members of supporting institutions.

Subscriptions, orders for numbers issued in the last three calendar years, and changes of address should be sent to Pacific Journal of Mathematics, P.O. Box 969, Carmel Valley, CA 93924, U.S.A. Old back numbers obtainable from Kraus Periodicals Co., Route 100, Millwood, NY 10546.

The Pacific Journal of Mathematics at P.O. Box 969, Carmel Valley, CA 93924 (ISSN 0030-8730) is published monthly except for July and August. Second-class postage paid at Carmel Valley, California 93924, and additional mailing offices. Postmaster: send address changes to Pacific Journal of Mathematics, P.O. Box 969, Carmel Valley, CA 93924.

PUBLISHED BY PACIFIC JOURNAL OF MATHEMATICS, A NON-PROFIT CORPORATION Copyright (C) 1991 by Pacific Journal of Mathematics 


\section{Pacific Journal of Mathematics}

Vol. 149, No. $2 \quad$ June, 1991

Manuel Alfaro Garcia, Mark Conger and Kenneth Hodges, The structure

of singularities in $\Phi$-minimizing networks in $\mathbf{R}^{2}$

Werner Balser, Dependence of differential equations upon parameters in

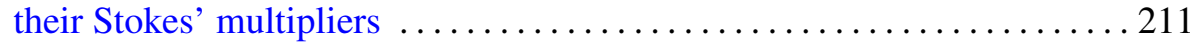

Enrico Casadio Tarabusi and Stefano Trapani, Envelopes of holomorphy

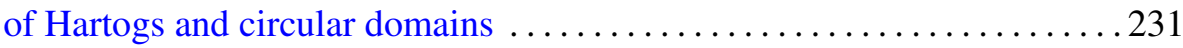

Hermann Flaschka and Luc Haine, Torus orbits in $G / P \ldots \ldots \ldots \ldots 251$

Gyo Taek Jin, The Cochran sequences of semi-boundary links .......... 293

Yasuyuki Kawahigashi, Cohomology of actions of discrete groups on

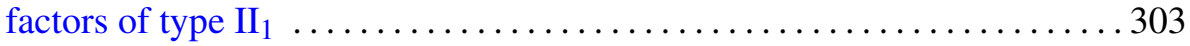

Ki Hyoung Ko and Lawrence Smolinsky, A combinatorial matrix in 3-manifold theory

W. B. Raymond Lickorish, Invariants for 3-manifolds from the combinatorics of the Jones polynomial

Peter Arnold Linnell, Zero divisors and group von Neumann algebras . . . . . 349

Bruce Harvey Wagner, Classification of essential commutants of abelian

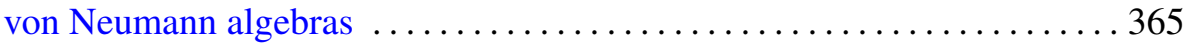

Herbert Walum, Multiplication formulae for periodic functions ..........383 